\title{
Rapid Eye Movement Sleep Behavior Disorder: Overview and Current Perspective
}

\author{
Muna Irfan $^{1} \cdot$ Michael J. Howell ${ }^{1}$
}

Published online: 14 April 2016

(C) Springer International Publishing AG 2016

\begin{abstract}
Rapid eye movement (REM) stage of sleep in normal circumstances is composed of vivid dream mentation associated with physiological skeletal muscle paralysis and thus, quiescence of motor activity. REM sleep behavior disorder (RBD) is a parasomnia characterized by disinhibition of motor control facilitating dream enactment behaviors. These nocturnal motoric behaviors due to lack of REM atonia range from benign limb movements to complex aggressive actions but are often manifested by violent, aggressive flailing, punching and kicking with vocalizations enacting action-filled hostile dreams. Polysomnographic evidence of increased electromyographic tone during REM with or without capturing clinical dream enactment together with careful history helps diagnose RBD. It is considered a prodromal feature of neurodegenerative alpha-synucleinopathies with high rate of eventual phenoconversion. The mainstay of treatment is to first ensure physical safety. When medications are necessary, options include either low-dose clonazepam, high-dose melatonin or both. Yearly surveillance with detailed neurological
\end{abstract}

This article is part of the Topical Collection on Parasomnias

Electronic supplementary material The online version of this article (doi:10.1007/s40675-016-0038-z) contains supplementary material, which is available to authorized users.

Muna Irfan

irfan007@umn.edu

Michael J. Howell

howel020@umn.edu

1 Department of Neurology, Minnesota Regional Sleep Disorders Center, Hennepin County Medical Center, University of Minnesota, Minneapolis, MN, USA evaluation is vital for early detection and eventual management of neurodegenerative disorder.

Keywords REM sleep behavior disorder .

Alpha-synucleinopathies $\cdot$ Dream enactment $\cdot$ REM sleep without atonia $\cdot$ REM parasomnia $\cdot$ Nocturnal motor activity . Oneirism

\section{Introduction}

A patient with rapid eye movement (REM) sleep behavior disorder (RBD) is often brought to medical attention when there has been injurious nocturnal behavior to patient or bed partner as a result of violent enactment of dreams.

In the following chapter, we will give a brief overview of epidemiology, pathogenesis and etiological association with special emphasis on alpha-synucleinopathies. We will also discuss nomenclature, demographics, clinical manifestations, differential diagnosis, polysomnographic findings and management strategies. The goal of treatment is to ensure safety of patient and bed partner and to ensure careful longitudinal follow-up of these patients. They should be serially evaluated for the development of subtle neurological signs or symptoms suggestive of neurodegeneration.

\section{Nomenclature}

\section{Rapid Eye Movement Sleep Without Atonia}

REM sleep without atonia (RSWA) is the neurophysiological finding of increased electromyographic (EMG) tone in REM sleep confirmed by polysomnogram (PSG). Some have proposed designating the term "subclinical RBD" for this group 
with implication that these patients will go on to develop dream enactment behavior, thus full spectrum of RBD but at this juncture, there is paucity of longitudinal follow-up of this population to arrive at this conclusion [1].

\section{Rapid Eye Movement Sleep Behavior Disorder}

RBD is a parasomnia characterized by recurrent dream enactment during REM sleep marked by neurophysiological substrate of increased EMG tone on PSG. For diagnosis of RBD, both clinical dream enactment and evidence of RSWA are required [2]. Some authors have used term clinically probable RBD if PSG testing for RSWA is not available but clinical history for dream enactment behavior is convincing.

\section{Idiopathic Rapid Eye Movement Sleep Behavior Disorder}

It refers to presence of RBD without any associated neurological disorder. While the term is still customarily used in literature, there has been growing opinion that RBD is a prodromal symptom in alpha-synucleinopathies, and hence it is not truly idiopathic $[1,3]$.

\section{Symptomatic Rapid Eye Movement Sleep Behavior Disorder}

This term denotes RBD in conjunction with a neurological disorder like an alpha-synuclein disorder [1,3], narcolepsy [1, $4,5]$, or paraneoplastic disorders [6, 7]. The expression secondary RBD is also used synonymously by other authors [1].

\section{Alpha-Synucleinopathies}

Disorders of neurodegeneration with alpha-synuclein pathology include Parkinson's disease, multiple system atrophy, dementia with Lewy bodies, and pure autonomic failure.

\section{Epidemiology and Demographics}

The estimated prevalence of RBD in general population is $0.5 \%[2,8]$ but significantly higher in elderly and specific neurodegenerative conditions. An elderly general population study cites PSG-proven RBD at frequency of $2 \%$ while RWSA in absence of clinical dream enactment of $5 \%$ [9].

In parkinsonian patients, the frequency of RBD is $30-54 \%$ [10-13]. The prevalence is higher in other synucleinopathies, such as $50-80 \%$ in dementia of Lewy body [14] and 80-95\% in multiple system atrophy $[15,16]$.

RBD in the young (less than 50 years old) is most commonly associated with narcolepsy or antidepressant use $[1,4$, 17]. RBD is reported in $60 \%$ of narcolepsy cases with a different phenotype, characterized by lack of the male preponderance that is seen in idiopathic RBD [8]. Of note, idiopathic
RBD can also occur in young adulthood predating the clinical symptoms of neurodegeneration by decades [18].

Despite the reported male predominance $(82-88 \%)[1,19$, 20], RBD is common in women. It is frequently undiagnosed in women due to less injurious dream enactment and due to the frequent lack of a bed partner to witness nocturnal behaviors due to longer life span of women compared to men [20,21]. Because of this, there has been some emerging evidence that RBD is more prevalent in females than is currently recognized [22].

Like Parkinson's disease, many cases of RBD are familial [8]. In addition, RBD is associated with various environmental, social, and behavioral factors. Patients with RBD are more likely to have a history of smoking, traumatic brain injury, pesticide exposure and fewer years of education [23].

\section{Etiopathogenesis}

Under normal circumstances, REM sleep motor atonia is the product of numerous brain pathways, most of which are localized to the pons. The critical structures in brain stem include "REMon" region consisting of precoeruleus (PC) and sublaterodorsal nucleus (SLD), and extended part of the ventrolateral preoptic nucleus (eVLPO), locus coeruleus (LC), laterodorsal tegmental nucleus (LDTN), pedunculopontine nucleus (PPN), and raphe nucleus (RN). REM-off region consists of the ventrolateral part of the periaqueductal grey matter (vl-PAG) and lateral pontine tegmentum (LPT) [1]. The loss of REM sleep atonia in typical RBD appears to be due to dysfunction of this on/off switch for control of REM sleep [24]. The actual circuitry is complex and various processes such as other sleep disorders, drugs, and structural lesions have been demonstrated to lead to RBD. The basic pathophysiological mechanism of REM sleep-related atonia is illustrated in schematic diagram (Fig. 1). Axons originate from "REM-on region" (mainly PC and SLD) and synapse on nucleus magnocellularis of medial medulla. Neurotransmitters released from the originating axons cause postsynaptic inhibition resulting in skeletal muscle atonia.

In RBD associated with narcolepsy, altered/decreased function of hypocretin pathways projecting from the lateral hypothalamus to the brainstem plays a causative role [4, 25]. Orexin secreted by lateral hypothalamus promotes state stability, and thus its deficiency causes REM-wake instability leading to REM dream mentation with excess motor activity $[5,26]$.

RBD is associated with several neurological disorders, as summarized in Table 1. The disorders with the strongest RBD link are represented by superscript "a" $[14,15,27]$.

\section{Alpha-Synucleinopathies}

The strong association between RBD and alphasynucleinopathies has been well established. The term encompasses the neurodegenerative disorders characterized by 


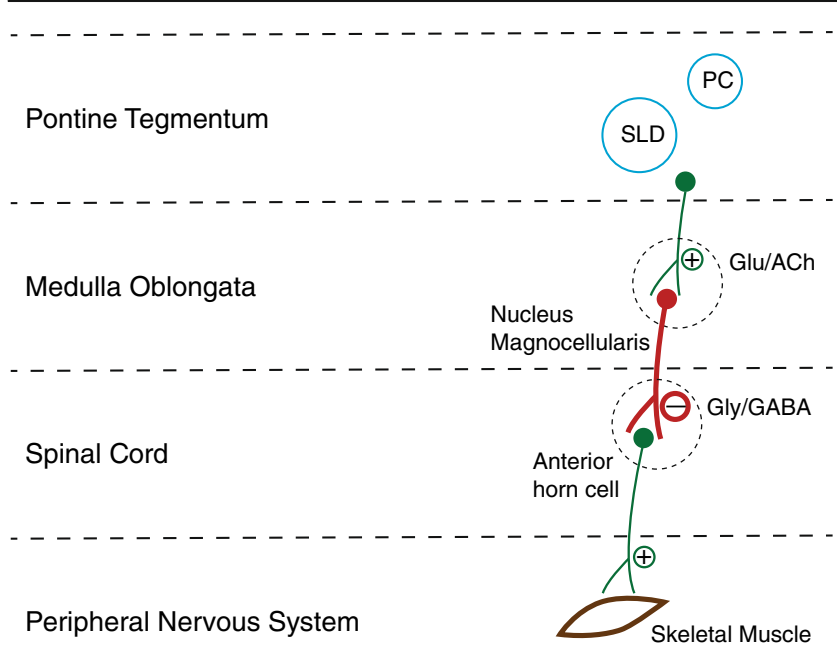

(a) REM Sleep Associated Atonia

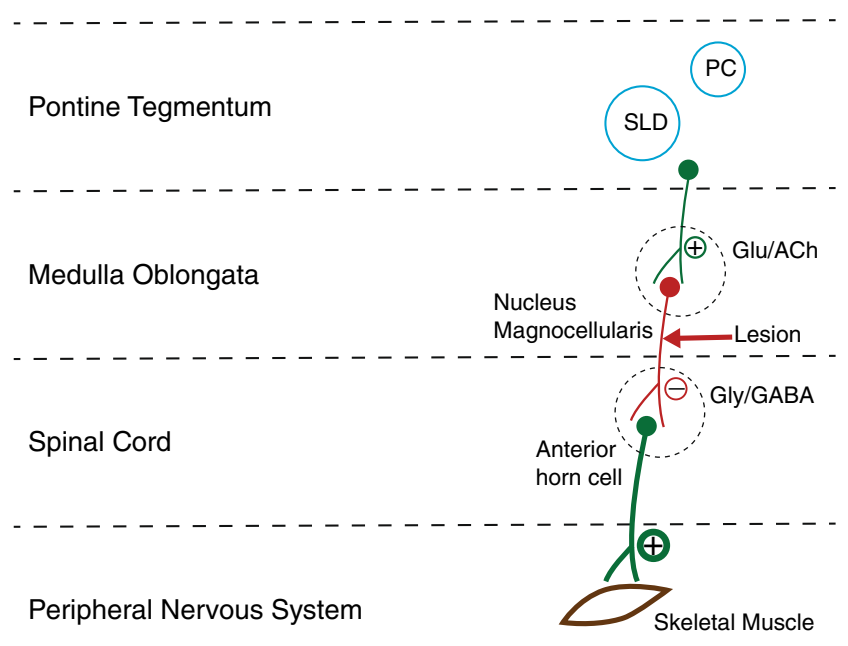

(b) Lack of REM Sleep Atonia

Fig. 1 a Schematic illustration of neural pathways mediating normal skeletal muscle atonia in REM sleep. b Disruption of this pathway leading to loss of REM-associated atonia and, thus, increasing tone. Glu glutamate, Ach acetylcholine, Gly glycine, GABA gamma aminobutyric acid, + excitatory neurotransmitter, - inhibitory neurotransmitter

pathologic alpha-synuclein deposits in central nervous system such as Parkinson's disease (PD), dementia of Lewy body (DLB), multiple system atrophy (MSA) and pure autonomic failure (PAF). These disorders are characterized by presence of Lewy bodies and aggregates of alpha synuclein and ubiquitin in neurons, which can be detected on autopsy [3, 28, 29]. Idiopathic RBD is a prodromal symptom of the disease, as it can predate the development of neurological disorder by decades $[1,18]$. The brainstem nuclei involved in REM sleep atonia generation are affected early in the disease process, thus facilitating RBD emergence before cortical pathology [30-32].

Several neuroimaging techniques have shown changes indicative of ongoing pathologic process in RBD such as transcranial ultrasound which has demonstrated substantia nigra hyperechogenicity [33] and nuclear imaging which has revealed reduced striatal dopamine transporters [34], dopaminergic innervation [35], and altered nigrostriatal and nigrocortical connectivity [36, 37]. All of these results are consistent with a developing dopaminergic deficit seen in disorders of parkinsonism.

Investigations have demonstrated unique metabolic network abnormalities in RBD as measured by $18 \mathrm{~F}$ fluorodeoxyglucose-positron emission tomography (FDGPET) and ethyl cysteinate dimer single positron emission tomography (ECD SPECT) which can predict ultimate conversion to clinical Parkinson's disease [38, 39].

MRI volumetric studies of RBD reveal grey matter reductions in the cerebellum, pons, and parahippocampus, similar to studies performed in DLB and MSA [40].

Evidence of wake and REM electroencephalographic (EEG) slowing has also been noted indicating cortical dysfunction $[41,42]$.

\section{Other Neurological Disorders}

RBD can also be associated with other neurodegenerative diseases in which disease pathology might topographically involve the pontine REM sleep generators. In contrast to alphasynuclein disorders, it is not a precursor in these conditions but occurs either coincidental or subsequent to development of other neurological symptoms [43]. Table 1 enlists various disorders such as amyloidopathies, tauopathies, TDP-43 proteinopathies, tri/tetranucleotide repeat disorders, genetic, congenital, and developmental conditions which have been reported to occur with RBD although at a much lower frequency [3, 44-47]. The one exception is spinocerebellar ataxia type 3 (SCA3), a trinucleotide repeat disorder of cerebellar and brainstem degeneration. The majority of patients with SCA-3 develop RBD.

Narcolepsy and RBD both represent dysfunction of awake REM state boundary control. The association of RBD with this disease is strongest in narcolepsy type 1 (narcolepsy with cataplexy) [5]. As discussed above, it represents unique phenotype, with equal gender distribution and less aggressive dream enactment movements [48].

\section{Medication-Associated Rapid Eye Movement Sleep Behavior Disorder}

Medication-associated RBD may be the most common form of RBD, especially among the young $[49,50]$. Approximately $10 \%$ of depressed patients exhibit increase in REM sleep motor tone after treatment with sertraline compared to $3 \%$ prior to treatment [51].

Several psychoactive drugs have been noted to precipitate or aggravate RSWA and RBD including tricyclic and tetracyclic antidepressants, monoamine oxidase (MAO) inhibitors, serotonin-specific reuptake inhibitors (SSRI) and 
Table 1 Neurological disorders associated with RBD

\begin{tabular}{|c|c|}
\hline ALPHA-SYNUCLEINOPATHY ${ }^{a}$ & GENETIC DISORDERS \\
\hline Parkinson's disease [30-54\%] & Wilsons disease \\
\hline Dementia with Lewy bodies [50-80 \%] & Pantothenate kinase-associated neurodegeneration \\
\hline \multicolumn{2}{|l|}{ Multiple system atrophy [80-95\%] } \\
\hline Pure autonomic failure & $\begin{array}{l}\text { CONGENITAL/DEVELOPMENTAL } \\
\text { DISORDERS }\end{array}$ \\
\hline AMYLOIDOPATHY & Moebius syndrome \\
\hline \multirow[t]{2}{*}{ Alzheimer's disease } & Smith-Magenis syndrome \\
\hline & Autism \\
\hline TAUOPATHY & Tourette syndrome \\
\hline \multicolumn{2}{|l|}{ Progressive supranuclear palsy } \\
\hline Guadeloupean parkinsonism & STRUCTURAL LESIONS \\
\hline Frontotemporal dementia & Multiple sclerosis \\
\hline \multirow[t]{2}{*}{ Amyotrophic lateral sclerosis } & Astrocytoma \\
\hline & Acoustic neuroma \\
\hline TRINUCLEOTIDE/TETRANUCLEOTIDE & Vascular malformations \\
\hline REPEAT DISORDERS & CNS vasculitis \\
\hline Spinocerebellar ataxia- $3^{\mathrm{a}}$ & Infarct \\
\hline \multicolumn{2}{|l|}{ Huntington's disease } \\
\hline Myotonic dystrophy type 2 & MEDICATION INDUCED ${ }^{\mathrm{a}}$ \\
\hline $\begin{array}{l}\text { OREXIN DEFICIENCY } \\
\text { Narcolepsy }\end{array}$ & $\begin{array}{l}\text { Tricyclic and tetracyclic antidepressants, } \\
\text { monoamine oxidase inhibitors, } \\
\text { selective serotonin inhibitors, serotonin } \\
\text { norepinephrine reuptake inhibitors }\end{array}$ \\
\hline $\begin{array}{l}\text { AUTOIMMUNE/INFLAMMATORY } \\
\text { DISORDERS }\end{array}$ & MEDICATION WITHDRAWAL (acute) \\
\hline Voltage-gated $\mathrm{K}$ antibody limbic encephalitis & Alcohol, barbiturate, amphetamine, cocaine \\
\hline \multicolumn{2}{|l|}{ Paraneoplastic cerebellar degeneration } \\
\hline \multicolumn{2}{|l|}{ Ma2 encephalitis } \\
\hline IgLON-5 parasomnia & \\
\hline
\end{tabular}

${ }^{\text {a }}$ Disorders with the strongest RBD link [14, 15, 27, 75] serotonin-norepinephrine reuptake inhibitors (SNRI), particularly venlafaxine, pentazocine, tramadol, and acetylcholinesterase inhibitors $[50,52,53]$. Drug-induced RBD cases tend to have high EMG tone predominantly in tibial leads [54].

Emerging evidence suggests that these medications may be unmasking a neurodegenerative disorder rather than causing de novo induction. According to one study, patients with antidepressant associated RBD also exhibited other prodromal symptoms like hyposmia, constipation, and subtle visual and motor findings [55]. Reduced striatal dopamine uptake as measured by (18) F-DOPA PET imaging has also been demonstrated in patients with medication-induced RBD [56]. These findings are intriguing and suggest that medicationinduced RBD may also be a prodromal syndrome to alphasynuclein degeneration; however, more studies are needed.

\section{Lesional Rapid Eye Movement Sleep Behavior Disorder}

A structural lesion localized to pontine centers governing REM sleep motor tone can also lead to RBD. Thus, cases of
RBD caused by vascular, neoplastic, demyelinating, vasculitic and traumatic insults have been reported [57, 58]. In such cases, cranial imaging reveals the structural pathology and topographic involvement of pontine tegmentum in most cases.

\section{Inflammatory/Autoimmune Rapid Eye Movement Sleep Behavior Disorder}

RBD can also occur with certain autoimmune and inflammatory conditions without any identifiable structural lesion. The most common association is with voltage-gated potassium antibody encephalopathies such as limbic encephalitis and Morvan's syndrome [59]. It has also been reported in other encephalitides like Ma-2 enchephalitis and paraneoplastic cerebellar degeneration [6, 7]. Importantly, these conditions respond favorably to immunotherapy. In these cases, reciprocal anatomical connections between limbic system and brainstem regions are presumed to affect REM sleep muscle atonia [28].

Recently, a unique neurological syndrome has been described in eight patients in Europe with antibodies to IgLON- 
5 , a type of neuronal cell adhesion molecule, suggesting a novel inflammatory mechanism. It is a distinctive condition with both nonrapid eye movement (NREM) and REM parasomnias, sleep-related breathing dysfunction, dysautonomia, bulbar, neurological dysfunction, and pathological features suggesting a tauopathy. Clinical deterioration, lack of response to immunosuppressive agents, and mortality were noted in all the cases. The exact role of IgLON-5 antibody in tau pathogenesis is unclear, and further investigation is warranted [60].

\section{Clinical Manifestations}

Clinical phenomenology of RBD is characterized by abnormal vocalizations and motoric behavior representing dream enactment.

Abnormal vocalizations in RBD tend to be loud suggesting unpleasant dreams. Shouting, hollering, screaming and swearing are fairly common and are often contradictory to the typically soft-spoken nature of the person during wakefulness. This helps in distinguishing from NREM parasomnias, where vocalization is more often of a benign conversational nature [3].

In RBD, the motor activity ranges on a wide spectrum from subtle hand movements (often referred to as hand babbling) to more dramatic, violent and seemingly purposeful actions such as punching, flailing, running, jumping out of bed, etc. [2]. These behaviors can prove to be injurious to the patients and their bed partners. Unlike NREM parasomnias, the duration of behaviors in RBD is typically brief with a rapid return to alertness on arousal.

The dream content often involves animals or people chasing or attacking the patient or their loved ones with patient almost always assuming role of the defender. Many patients are able to recount the content of their dreams upon being awakened. Individuals with RBD can often recall vivid details of the nightmares for days and sometimes for weeks or years [3].

The frequency of dream enactment behaviors (DEB) also varies widely from several times every night to one night per month or less. Some appear to exhibit clustering without any known trigger.

Since REM sleep periods in latter half of the night are longer and with greater phasic REM activity, dream enactment is more pronounced in second part of the night [1].

\section{Diagnostic Criteria (ICSD-3)}

According to third edition of the International Classification of Sleep Disorders, the diagnosis of RBD requires fulfilling following criteria:

1. Recurrent nocturnal episodes of vocalizations and/or complex motoric behaviors consistent with dream enactment.
2. These behaviors are evidenced by in-laboratory PSG to occur in REM sleep or presumed to occur so based on clinical history.

3. Evidence of RSWA is obtained on in-laboratory PSG.

4. Sleep-related disturbance is not explained by another sleep or mental health disorder, medication or substance use

\section{Polysomnographic Characteristics}

According to the American Academy of Sleep Medicine, the polysomnographic features of RBD are characterized by excessive

1. Tonic activity in REM sleep in chin EMG and/or

2. Phasic activity during REM sleep in either chin or limb activity

Tonic activity (sustained muscle activity) is demonstrated by an epoch of REM sleep with at least $50 \%$ of the duration of the epoch having chin EMG amplitude greater than the minimum amplitude noted in NREM sleep.

Phasic activity (excessive transient muscle activity) is demonstrated in submental or limb EMG when a 30 -second epoch divided into ten sequential 3second mini-epochs has at least five $(50 \%)$ miniepochs of bursts of transient muscle activity. These bursts are $0.1-0.5$ second in duration and at least four times greater in amplitude than background EMG activity [2]. Figure 2 demonstrates neurophysiologic findings of RBD on polysomnographic recording.

REM sleep atonia index (RAI) is a quantitative RSWA metric ranging from 0.0 to 1.0 (the lower the number, the greater the amount of REM sleep motor activity). It has been shown to demonstrate reasonable night-to-night consistency [61]. In order to standardize and objectively quantify excessive motor activity, computer-assisted scoring methods have been developed and an abnormal REM atonia index is defined as $(<0.88)[54,62,63]$.

\section{Diagnostic Tools}

Several screening surveys are available to help identify RBD among general and disease populations. These tools share many similarities: they all inquire about the presence of dream enactment, and thus all have relatively similar sensitivities and specificities. A bed partner, if available, increases their yield and efforts should be made to include their observations as patients are often unaware of their behaviors. While an in-laboratory 


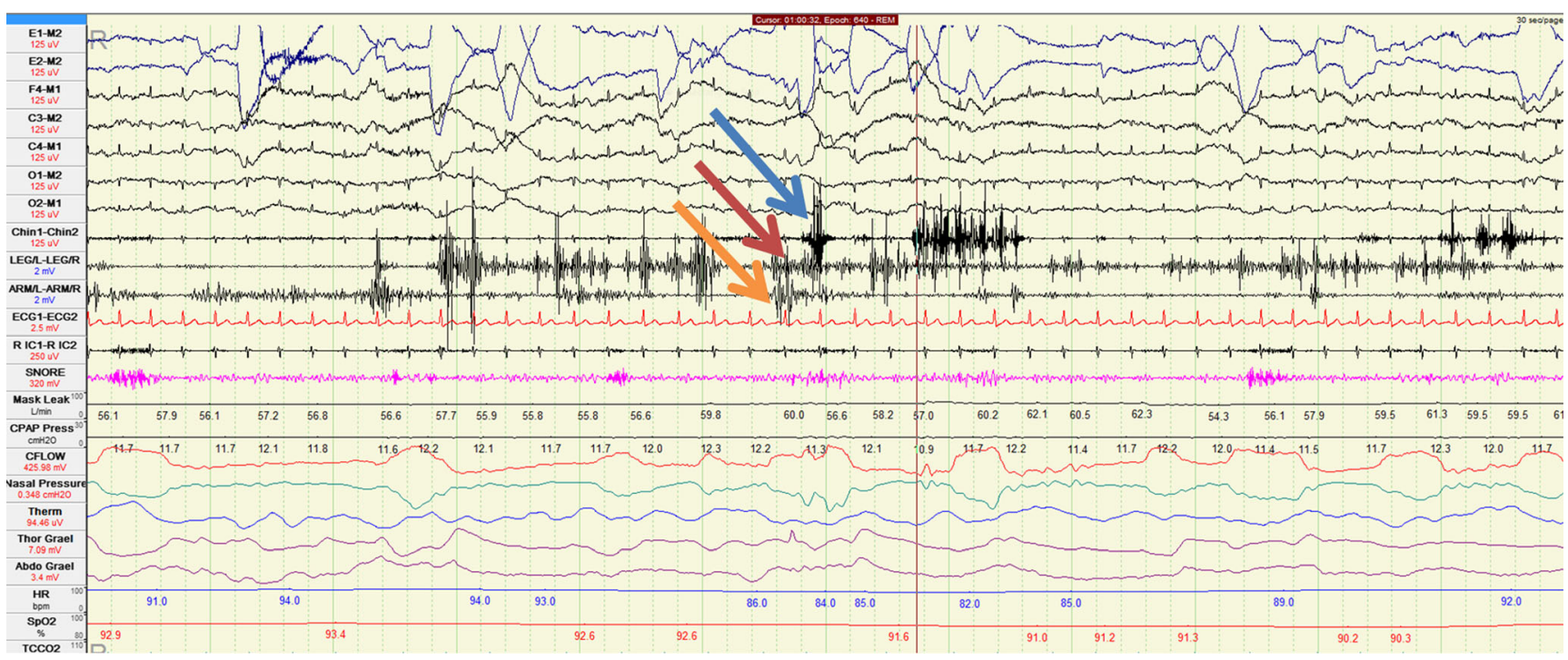

Fig. 2 Thirty-second epoch of polysomnogram showing increased electromyographic tone in submental (Chin1-chin2) denoted by blue arrow, in lower limb (left leg to right leg) denoted by red arrow, and in upper limb (left arm to right arm) derivations denoted by orange arrow

sleep study is needed for ICSD-3 diagnosis, initial PSG can miss up to $20 \%$ of RBD cases [64] which could explain the relatively low specificity of the clinical tools noted below.

The RBD Questionnaire-Hong Kong (RBDQ-HK) is a validated 13-item measure with higher scores indicating greater severity. It has a positive predictive value of $86 \%$ and a negative predictive value of $83 \%$ [65].

The REM Sleep Behavior Disorder Screening Questionnaire (RBDSQ) is a ten-item screen, and a score greater than 4 has a sensitivity of $90 \%$ and a specificity of $87 \%$ [66].

Interestingly, a single RBD question-based Mayo Sleep Questionnaire (MSQ) demonstrated high diagnostic sensitivity and specificity of 98 and $74 \%$ [67]. The referenced informant question is "Have you ever seen the patient appear to 'act out his/her dreams' while sleeping? (punched or flailed arms in the air, shouted, or screamed)."

Another survey, the RBD Single-Question Screen (RBD1Q) showed sensitivity of $94 \%$ and specificity $87 \%$ [68].

Innsbruck RBD inventory, a five-item-based questionnaire, has sensitivity and specificity of 91.4 and $86 \%$, respectively [69].

\section{Differential Diagnosis}

The other sleep-related conditions that can present like RBD include NREM parasomnias such as sleep walking, confusional arousals, and sleep terrors. These can be teased out by meticulous historical account. These disorders of arousal will occur in first half of the night, without any recollection of the event or dream mentation. Careful determination of nocturnal behaviors especially through collateral history by a caregiver or bed partner is crucial. Some of the factors differentiating
RBD from a NREM parasomnia include initial presentation in middle age or the elderly, present in second half of the sleep period with eyes closed, dream recall, and low arousal threshold (the patients are easily woken up). Behaviors mostly occur in bed with jerky movements occurring in bursts with sudden jumping off the bed while enacting dreams. When sleep walking occurs, it is mostly in context of rushed movement of escape or rescue resulting in fall or throwing themselves against physical barriers [70]. Other conditions that lead to nocturnal arousals mimicking RBD are obstructive sleep apnea, periodic limb movements of sleep and gastroesophageal reflux $[1,3]$. Pseudo-RBD comprises of RBD-like symptoms in patients who have obstructive sleep apnea. It is manifested by abnormal behavior occurring with arousal at the end of obstructive apneic event [71]. Nocturnal frontal lobe epilepsy is also in the differential which is marked by recurrent, stereotypical hypermotor seizures typically during NREM sleep. When seizures are suspected, an extended EEG montage should be employed during the in-laboratory PSG. Other conditions that can manifest as abnormal nocturnal behaviors included rhythmic movement disorder and psychogenic dissociative disorder.

On occasion, patients with RBD also demonstrate a NREM disorder of arousal as well such as sleepwalking. This is parasomnia overlap disorder, a subtype of RBD in the ICSD3. Patients with parasomnia overlap disorder are often younger than patients with idiopathic RBD and typically respond well to conventional sleepwalking and RBD therapies [72, 73].

While polysomnography is often helpful in diagnosing other parasomnias, RBD is the only condition that requires polysomnography for ICSD-3 criteria [2]. Table 2 summarizes common sleep conditions mimicking RBD. 
Table 2 Conditions included in differential diagnosis of RBD

Conditions mimicking RBD

Sleepwalking

Sleep terrors

Obstructive sleep apnea "pseudo-RBD"

Nocturnal seizures

Rhythmic movement disorder

Posttraumatic stress disorder

Complex nocturnal visual hallucinations

Periodic movements of sleep

Psychogenic dissociative disorder

\section{Treatment}

Foremost goal of management of RBD is ensuring safety of the patient and bed partner. There should be strong emphasis on making sleeping environment safe (level A evidence according to treatment guidelines [74]). Spouses and other bed partners should sleep separately. Firearms, as well as sharp potentially injurious objects, should be removed from the bedroom. Other options include the following: using a bed with padded rails, a mattress on the floor, and/or a sleeping bed to limit potential injury.

The next steps should include the elimination and substitution of aggravating drugs (if feasible) and treating any other exacerbating sleep-related conditions like OSA.

Pharmacological treatment options are discussed below.

\section{Clonazepam}

It has been the most widely prescribed agent historically, which is used in 0.5-1-mg doses with response rate of approximately $90 \%$ (level B) $[25,73,74]$. The mechanism of action is not clearly understood and has minimal effect on EMG tone in REM sleep.

However, the results of longitudinal studies with clonazepam range from sustained benefit to incremental dose escalation and treatment failure [3, 27, 75, 76]. According to one study, $58 \%$ of patients on clonazepam reported significant adverse effects, likely owed to its long half-life, leading to dose reduction or cessation in $50 \%$ [76]. The adverse effects of confusion, sedation, respiratory depression, and cognitive and gait disturbances can be dangerous in RBD patients with alpha-synucleinopathies, and thus, careful monitoring is advised (level B) [74].

2. Melatonin

It has been increasingly used in RBD as a first-line agent in a dose range from 3 to $15 \mathrm{mg}$ (level B) $[3,74]$. Although the mechanism of action is unclear, melatonin may restore RBD-related desynchronization of circadian rhythms. In contrast to clonazepam, it is also shown to decrease the number of REM epochs without atonia and decrease both phasic and tonic motor activities [77, 78]. Also, recent studies have shown favorability due to better side effect profile, with reduced falls and injuries when compared to clonazepam $[74,79]$. This becomes especially relevant in RBD associated with neurodegeneration as these patients are vulnerable due to low cognitive reserve. Most common side effects include morning headaches, mild sleepiness and delusions/hallucinations. Ramelteon, a melatonin agonist, has also been reported to treat RBD in two cases [80].

According to expert consensus, both clonazepam and melatonin are reasonable first-line therapies [74].

3. Pramipexole

This dopamine agonist can also be used to treat RBD especially in cases associated with periodic limb movements (PLMS). A study of 14 patients demonstrated improvement in RBD in $80 \%$ cases with reduction in REM density and PLM index but no change in RSWA [81].

4. Other agents

There is some data suggesting use of acetylcholinesterase inhibitors like rivastigmine especially in RBD with associated cognitive impairment [Brunetti et al. 2014]. Other agents with efficacy based on limited case reports include imipramine, desipramine, carbamazepine, levodopa, Yi-Gan San, sodium oxybate, triazolam, zopiclone, quetiapine, clozapine, and cannabidiol $[3,74,82]$.

\section{Bed Alarm Therapy}

Medication refractory RBD is a challenging and potentially life-threatening condition. Escaping the bed during dream enactment may result in serious trauma. Fortunately, the brain is responsive to auditory sound processing during REM sleep [83]. This principle applied through a customized pressure sensing bed alarm can deliver a calming message (usually from a family member) at the onset of dream enactment [84]. When the patient arises from the bed, the pressure pad triggers a voice recording such as "Bob you are having a dream. Lay back down." The patient then lies down, the recording shuts off and the patient returns to REM sleep.

\section{Prognosis and Phenoconversion}

Physically aggressive dream enactment behavior in RBD can pose a serious threat to the patient or bed partner. Trauma incurred can range from minor injuries to subdural hematoma, shoulder dislocation, cervical fracture, pelvic fracture, and lacerations severing arteries, tendons, and nerves. When a 
bed partner is involved, injuries can have legal ramifications [3]. Environmental adaptation and timely pharmacotherapy can prevent these undesirable consequences.

As idiopathic RBD is a prodromal syndrome, a diagnosis dramatically increases a patient's risk of developing PD or other alpha-synuclein syndrome. Two longitudinal cohorts of RBD have shown approximately $50 \%$ conversion rate in the first 10 years [30-32]. Ultimately, however, nearly all (81-91\%) surviving RBD patients demonstrate phenoconversion $[18,85]$.

RBD patients may be stratified by assessing other premotor symptoms and biomarkers of alphasynucleinopathies, such as hyposmia and constipation which, when present, help identify individuals at high risk of disease conversion [32, 86]. Imaging modalities for dopamine dysfunction, such as dopamine transporter scan DaTscan and impaired autonomic regulation, such as MIBG cardiac scintigraphy, while useful in research studies, do not have established a role in clinical context [3].

While research on idiopathic RBD and predictive biomarkers continues to be an area of intense effort, the responsibility of discussion of the risk of phenoconversion lies with the treating physician. Also in current age, where most of the patients are computer savvy, they should receive counseling from appropriate medical sources rather than obtaining information from random social media. It is considered standard of practice to discuss the risk of conversion to neurodegenerative disorder with the patient at an appropriate time in a therapeutic relationship. The need for yearly surveillance with neurological exam should be discussed with the patient in attempt for early detection and symptomatic management. It also allows patient autonomy and provides them with an opportunity to make informed decision about their medical care and alleviate unnecessary distress to the family later on [1].

\section{Conclusion}

$\mathrm{RBD}$ is an intriguing disorder characterized by dream enactment and increased REM sleep motor tone on PSG. It's diagnosis has several clinical implications including sleeprelated injury to patients and bed partners and predicting the development of alpha-synuclein neurodegenerative disorder sometimes decades into the future. Management includes environmental modification or pharmacotherapy with melatonin and/or clonazepam.

Clinically, it is important to frequently screen RBD patients for the development of subtle neurological symptoms, although at this time, disease-modifying therapies have not yet been proven. RBD detection is important as it allows clinicians to monitor and manage the patients' neurodegenerative process. Importantly, an international consortium of RBD investigators, the International RBD study Group (IRBDSG) has been established and is developing clinical trials for neuroprotection [87].

\section{Compliance with Ethical Standards}

Conflict of Interest The authors declare that they have no conflict of interest.

Human and Animal Rights and Informed Consent This article does not contain any studies with human or animal subjects performed by any of the authors.

\section{References}

1. Boeve BF. REM sleep behavior disorder: updated review of the core features, the REM sleep behavior disorder-neurodegenerative disease association, evolving concepts, controversies, and future directions. Ann N Y Acad Sci. 2010;1184:15-54.

2. American Academy of Sleep Medicine. International Classification of Sleep Disorders: Diagnostic and Coding Manual. (AASM ICSD-3). 3rd ed. Westchester: American Academy of Sleep Medicine; 2014.

3. Howell MJ, Schenck CH. Rapid eye movement sleep behavior disorder and neurodegenerative disease. JAMA Neurol. 2015.

4. Nightingale $S$ et al. The association between narcolepsy and REM behavior disorder (RBD). Sleep Med. 2005;6(3):253-8.

5. Nevsimalova S, Pisko J, Buskova J, et al. Narcolepsy: clinical differences and association with other sleep disorders in different age groups. J Neurol. 2013;260(3):767-75.

6. Compta Y, Iranzo A, Santamaría J, Casamitjana R, Graus F. REM sleep behavior disorder and narcoleptic features in anti-Ma2associated encephalitis. Sleep. 2007;30(6):767-9.

7. Vale TC, Fernandes do Prado LB, Fernandes do Prado G, Barsottini OG, Pedroso JL. Sleep rapid eye movement-sleep behavior disorder in paraneoplastic cerebellar degeneration: improvement with immunotherapy. 2015.

8. Dauvilliers Y, Postuma RB, Ferini-Strambi L, et al. Family history of idiopathic REM behavior disorder: a multicenter case-control study. Neurology. 2013;80(24):2233-5.

9. Kang SH, Yoon IY, Lee SD, et al. REM sleep behavior disorder in the Korean elderly population: prevalence and clinical characteristics. Sleep. 2013;36(8):1147-52.

10. Gagnon JF, Bedard MA, Fantini ML, et al. REM sleep behavior disorder and REM sleep without atonia in Parkinson's disease. Neurology. 2002;59(4):585-9.

11. Plomhause L, Dujardin K, Duhamel A, et al. Rapid eye movement sleep behavior disorder in treatment-naïve Parkinson disease patients. Sleep Med. 2013;14(10):1035-7.

12. Poryazova R, Oberholzer M, Baumann CR, Bassetti CL. REM sleep behavior disorder in Parkinson's disease: a questionnairebased survey. J Clin Sleep Med. 2013;9(1):55-9A.

13. Yoritaka A, Ohizumi H, Tanaka S, Hattori N. Parkinson's disease with and without REM sleep behaviour disorder: are there any clinical differences? Eur Neurol. 2009;61(3):164-70.

14. Boeve BF, Silber MH, Ferman TJ. REM sleep behavior disorder in Parkinson's disease and dementia with Lewy bodies. J Geriatr Psychiatry Neurol. 2004;17(3):146-57.

15. Plazzi G, Corsini R, Provini F, et al. REM sleep behavior disorder in multiple system atrophy. Neurology. 1997;48:1094-7. 
16. Palma J-A, Fernandez-Cordon C, Coon EA, et al. Prevalence of REM sleep behavior disorder in multiple system atrophy: a multicenter study and meta-analysis. Clin Auton Res Off J Clin Auton Res Soc. 2015;25(1):69-75. doi:10.1007/s10286-015-0279-9.

17. Lloyd R, Tippmann-Peikert M, Slocumb N, Kotagal S. Characteristics of REM sleep behavior disorder in childhood. J Clin Sleep Med. 2012;8(2):127-31.

18. Schenck CH, Boeve BF, Mahowald MW. Delayed emergence of a parkinsonian disorder or dementia in $81 \%$ of older men initially diagnosed with idiopathic rapid eye movement sleep behavior disorder: a 16-year update on a previously reported series. Sleep Med. 2013;14(8):744-8.

19. Wing YK, Lam SP, Li SX, et al. REM sleep behaviour disorder in Hong Kong Chinese: clinical outcome and gender comparison. J Neurol Neurosurg Psychiatry. 2008;79(12):1415-6.

20. Bodkin CL, Schenck CH. Rapid eye movement sleep behavior disorder in women: relevance to general and specialty medical practice. J Womens Health (Larchmt). 2009;18(12):1955-63.

21. Zhou J, Zhang J, Li Y, et al. Gender differences in REM sleep behavior disorder: a clinical and polysomnographic study in China. Sleep Med. 2015;16:414-8.

22. Bjornara KA, Dietrichs E, Toft M. REM sleep behavior disorder in Parkinson's disease - is there a gender difference. Parkinsonism Relat Disord. 2013;19(1):120-2.

23. Postuma RB, Montplaisir JY, Pelletier A, et al. Environmental risk factors for REM sleep behavior disorder: a multicenter case-control study. Neurology. 2012;79(5):428-34. doi:10.1212/WNL. 0b013e31825dd383.

24. Lu J, Sherman D, Devor M, Saper C. A putative flip-flop switch for control of REM sleep. Nature. 2006;441:589-94.

25. Schenck $\mathrm{CH}$, Mahowald MW. Motor dyscontrol in narcolepsy: rapid-eye-movement (REM) sleep without atonia and REM sleep behavior disorder. Ann Neurol. 1992;32(1):3-10.

26. Hsieh KC, Nguyen D, Siegel JM, Lai YY. New pathways and data on REM sleep behaviour disorder in a rat model. Sleep Med. 2013;14(8):719-28.

27. Schenck CH, Bundlie SR, Mahowald MW. Delayed emergence of a parkinsonian disorder in $38 \%$ of 29 older men initially diagnosed with idiopathic rapid eye movement sleep behavior disorder. Neurology. 1996;46(2):388-93.

28. Iranzo A, Santamaria J, Tolosa E. The clinical and pathophysiological relevance of REM sleep behavior disorder in neurodegenerative diseases. Sleep Med Rev. 2009;13(6):385-401.

29. Hu Y, Yu SY, Zuo LJ, et al. Parkinson disease with REM sleep behavior disorder: features, alpha-synuclein, and inflammation. Neurology. 2015;84:888-94.

30. Schenck $\mathrm{CH}$, Mahowald MW. Longitudinal follow up of the original REM sleep behavior disorder cohort. Sleep. 2003;26:A316.

31. Iranzo A, Molinuevo JL, Santamaria J, et al. Rapid-eye-movement sleep behavior disorder as an early marker for a neurodegenerative disorder: a descriptive study. Lancet Neurol. 2006;5(7):572-7.

32. Postuma RB, Gagnon JF, Vendette M, Fantini ML, MassicotteMarquez J, Montplaisir J. Quantifying the risk of neurodegenerative disease in idiopathic REM sleep behavior disorder. Neurology. 2009;72(15):1296-300.

33. Shin HY, Joo EY, Kim ST, Dhong HJ, Cho JW. Comparison study of olfactory function and substantia nigra hyperechogenicity in idiopathic REM sleep behavior disorder, Parkinson's disease and normal control. Neurol Sci. 2013;34(6):935-40.

34. Eisensehr I, Linke R, Tatsch K, et al. Increased muscle activity during rapid eye movement sleep correlates with decrease of striatal presynaptic dopamine transporters. IPT and IBZM SPECT imaging in subclinical and clinically manifest idiopathic REM sleep behavior disorder, Parkinson's disease, and controls. Sleep. 2003;26(5):507-12.
35. Albin RL, Koeppe RA, Chervin RD, et al. Decreased striatal dopaminergic innervation in REM sleep behavior disorder. Neurology. 2000;55(9):1410-2.

36. Mossa EP, Niccoli Asabella A, Iuele F, Stabile Ianora AA, Giganti M, Rubini G. Striatal dopamine transporter levels in patients with REM sleep behavior disorder: assessment with 123I-FP-CIT SPECT. [Article in Italian]. Recenti Prog Med. 2012;103(11):500-4.

37. Ellmore TM, Castriotta RJ, Hendley KL, et al. Altered nigrostriatal and nigrocortical functional connectivity in rapid eye movement sleep behavior disorder. Sleep. 2013;36(12):1885-92.

38. Holtbernd F, Gagnon JF, Postuma RB, et al. Abnormal metabolic network activity in REM sleep behavior disorder. Neurology. 2014;82(7):620-7.

39. Wu P, Yu H, Peng S, et al. Consistent abnormalities in metabolic network activity in idiopathic rapid eye movement sleep behavior disorder. Brain. 2014;137:3122-8.

40. Hanyu H, Inoue Y, Sakurai H, et al. Voxel-based magnetic resonance imaging study of structural brain changes in patients with idiopathic REM sleep behavior disorder. Parkinsonism Relat Disord. 2012;18(2):136-9.

41. Fantini ML, Gagnon JF, Petit D, et al. Slowing of electroencephalogram in rapid eye movement sleep behavior disorder. Ann Neurol. 2003;53(6):774-80.

42. Massicotte-Marquez J, Carrier J, Decary A, et al. Slow-wave sleep and delta power in rapid eye movement sleep behavior disorder. Ann Neurol. 2005;57(2):277-82.

43. Boeve BF, Silber MH, Ferman TJ, Lin SC, et al. Clinicopathologic correlations in 172 cases of rapid eye movement sleep behavior disorder with or without a coexisting neurologic disorder. Sleep Med. 2013;14(8):795-806.

44. Tribi CG, Bor-Seng-Shu E, Trindade MC, Lucato LT, Teixeira MJ, Barbosa ER. Wilsons disease presenting as rapid eye movement sleep behavior disorder: a possible window to early treatment. Arq Neuropsiquiatr. 2014;72:653-8.

45. Chokroverty S, Bhat S, Rosen D, Farheen A. REM behavior disorder in myotonic dystrophy type 2. Neurology. 2012;78(24):2004.

46. Nomura T, Inoue Y, Takigawa H, Nakashima K. Comparison of REM sleep behaviour disorder variables between patients with progressive supranuclear palsy and those with Parkinson's disease. Parkinsonism Relat Disord. 2012;18(4):394-6.

47. Ebben MR, Shahbazi M, Lange DJ, Krieger AC. REM behavior disorder associated with familial amyotrophic lateral sclerosis. Amyotroph Lateral Scler. 2012;13(5):473-4.

48. Dauvilliers $\mathrm{Y}$ et al. Rapid eye movement sleep behavior disorder and rapid eye movement sleep without atonia in narcolepsy. Sleep Med. 2013;14(8):775-81.

49. Teman PT, Tippmann-Peikert M, Silber MH, Slocumb NL, Auger RR. Idiopathic rapid-eye-movement sleep disorder: associations with antidepressants, psychiatric diagnoses, and other factors, in relation to age of onset. Sleep Med. 2009;10(1):60-5.

50. Ju YE, Larson-Prior L, Duntley S. Changing demographics in REM sleep behavior disorder: possible effect of autoimmunity and antidepressants. Sleep Med. 2011;12(3):278-83.

51. Zhang B, Hao Y, Jia F, et al. Sertraline and rapid eye movement sleep without atonia: an 8 week, open-label study of depressed patients. Prog Neuropsychopharmacol Biol Psychiatry. 2013;47:85-92.

52. Hoque R, Chesson Jr AL. Pharmacologically induced/exacerbated restless legs syndrome, periodic limb movements of sleep, and REM behavior disorder/REM sleep without atonia: literature review, qualitative scoring, and comparative analysis. J Clin Sleep Med. 2010;6(1):79-83.

53. Frauscher B, Jennum P, Ju YE, et al. Comorbidity and medication in REM sleep behavior disorder: a multicenter case-control study. Neurology. 2014;82(12):1076-9. 
54. McCarter SJ, St Louis EK, Duwell EJ, et al. Diagnostic thresholds for quantitative REM sleep phasic burst duration, phasic and tonic muscle activity, and REM atonia index in REM sleep behavior disorder with and without comorbid obstructive sleep apnea. Sleep. 2014;37:1649-62.

55. Postuma RB, Gagnon JF, Pelletier A, Montplaisir J. Prodromal autonomic symptoms and signs in Parkinson's disease and dementia with Lewy bodies. Mov Disord. 2013;28(5):597-604.

56. Wing YK, Lam SP, Zhang J, et al. Reduced striatal dopamine transmission in REM sleep behavior disorder comorbid with depression. Neurology. 2015;84:516-22.

57. Tang WK, Hermann DM, Chen YK, et al. Brainstem infarcts predict REM sleep behavior disorder in acute ischemic stroke. BMC Neurol. 2014;14:88.

58. McCarter SJ et al. Neuroimaging-evident lesional pathology associated with REM sleep behavior disorder. Sleep Med. 2015;16(12):1502-10.

59. Cornelius JR, Pittock SJ, McKeon A, Lennon VA, Aston PA, Josephs KA, et al. Sleep manifestations of voltage-gated potassium channel complex autoimmunity. Arch Neurol. 2011;68(6):733-8.

60. Sabater L, Gaig C, Gelpi E, et al. A novel non-rapid-eye movement and rapid-eye-movement parasomnia with sleep disordered breathing associated with antibodies to IgLON5: a case series, characterization of the antigen, and post-mortem study. Lancet Neurol. 2014;13:575-86.

61. Bolitho SJ, Naismith SL, Terpening Z, et al. Investigating the nightto-night variability of REM without atonia in Parkinson's disease. Sleep Med. 2015;16:190-3.

62. Ferri R, Gagnon JF, Postuma RB, et al. Comparison between and automatic and visual scoring method of the chin muscle tone during rapid eye movement sleep. Sleep Med. 2014;15(6):661-5.

63. Frauscher B, Gabelia D, Biermayr M, et al. Validation of an integrated software for the detection of rapid eye movement sleep behavior disorder. Sleep. 2014;37:1663-71.

64. Montplaisir J, Gagnon JF, Fantini ML, et al. Polysomnographic diagnosis of idiopathic REM sleep behavior disorder. Mov Disord. 2010;25(13):2044-51.

65. Li SX, Wing YK, Lam SP, et al. Validation of a new REM sleep behavior disorder questionnaire (RBDQ-HK). Sleep Med. 2010;11(1):43-8.

66. Stiasny-Kolster K, Sixel-Doring F, Trenkwalder C, et al. Diagnostic value of the REM sleep behavior disorder screening questionnaire in Parkinson's disease. Sleep Med. 2015;16:186-9.

67. Boeve BF, Molano JR, Ferman TJ, et al. Validation of the Mayo Sleep Questionnaire to screen for REM sleep behavior disorder in a community based sample. J Clin Sleep Med. 2013;9(5):475-80.

68. Postuma RB, Arnulf I, Hogl B, et al. A single-question screen for rapid eye movement sleep behavior disorder: a multicenter validation study. Mov Disord. 2012;27(7):913-6.

69. Frauscher B et al. Validation of the Innsbruck REM sleep behavior disorder inventory. Mov Disord. 2012;27(13):1673-8

70. Uguccioni G, Golmard JL, de Fontreaux AN, Leu-Semenescu S, Brion A, Arnulf I. Fight or flight. Dream content during sleepwalking/sleep terrors vs. rapid eye movement sleep behavior disorder. Sleep Med. 2013;14(5):391-8.

71. Iranzo A, Santamaria J. Severe obstructive sleep apnea/hypopnea mimicking REM sleep behavior disorder. Sleep. 2005;28(2):203-6.
72. Schenck CH, Boyd JL, Mahowald MW. A parasomnia overlap disorder involving sleepwalking, sleep terrors, and REM sleep behavior disorder in 33 polysomnographically confirmed cases. Sleep. 1997;20(11):972-81.

73. Olson EJ, Boeve BF, Silber MH. Rapid eye movement sleep behaviour disorder: demographic, clinical and laboratory findings in 93 cases. Brain. 2000;123(Pt 2):331-9.

74. Aurora RN, Zak RS, Maganti RK, Auerbach SH, Standards of Practice Committee; American Academy of Sleep Medicine, et al. Best practice guide for the treatment of REM sleep behavior disorder (RBD). J Clin Sleep Med. 2010;6(1):85-95.

75. Nardone R, Bergmann J, Brigo F, et al. Functional evaluation of central cholinergic circuits in patients with Parkinson's disease and REM sleep behavior disorder: a TMS study. J Neural Transm. 2013;120(3):413-22.

76. Anderson KN, Shneerson JM. Drug treatment of REM sleep behavior disorder: the use of drug therapies other than clonazepam. J Clin Sleep Med. 2009;5(3):235-9.

77. Kunz D, Mahlberg R. A two-part, double-blind, placebo-controlled trial of exogenous melatonin in REM sleep behaviour disorder. J Sleep Res. 2010;19(4):591-6.

78. McGrane I, Leung J, St. Louis E, Boeve B. Melatonin therapy for REM sleep behavior disorder: a critical review of evidence. Sleep Med. 2015;16(1):19-26.

79. McCarter SJ, Boswell CL, St Louis EK, et al. Treatment outcomes in REM sleep behavior disorder. Sleep Med. 2013;14(3):237-42.

80. Nomura T, Kawase S, Watanabe Y, Nakashima K. Use of ramelteon for the treatment of secondary REM sleep behavior disorder. Intern Med. 2013;52(18):2123-6.

81. Sasai T, Inoue Y, Matsuura M. Effectiveness of pramipexole, a dopamine agonist, on rapid eye movement sleep behavior disorder. Tohoku J Exp Med. 2012;226(3):177-81.

82. Chagas MH, Eckeli AL, Zuardi AW, et al. Cannabidiol can improve complex sleep-related behaviors associated with rapid eye movement sleep behavior disorder in Parkinson's disease patients: a case series. J Clin Pharm Ther. 2014;39:564-6.

83. Atienza M, Cantero JL. Complex sound processing during human REM sleep by recovering information from long-term memory as revealed by the mismatch negativity $(\mathrm{MMN})$. Brain Res. 2001;901(1-2):151-60.

84. Howell MJ, Arneson PA, Schenck CH. A novel therapy for REM sleep behavior disorder (RBD). J Clin Sleep Med. 2011;7(6):639-44A.

85. Iranzo A, Fernandez-Arcos A, Tolosa E, et al. Neurodegenerative disorder risk in idiopathic REM sleep behavior disorder: study in 174 patients. PLoS ONE. 2014;9(2), e89741.

86. Postuma RB, Gagnon JF, Bertrand JA, et al. Parkinson risk in idiopathic REM sleep behavior disorder: preparing for neuroprotective trials. 2015 84:1104-13.

87. Schenck CH, Montplaisir JY, Frauscher B, et al. Rapid eye movement sleep behavior disorder: devising controlled active treatment studies for symptomatic and neuroprotective therapy-a consensus statement from the International Rapid Eye Movement Sleep Behavior Disorder Study Group. Sleep Med. 2013;14(8):795806. doi:10.1016/j.sleep.2013.02.016. 